TITLE:

\title{
Noise-bias compensation in physical-parameter system identification under microtremor input
}

$\operatorname{AUTHOR}(\mathrm{S}):$

Yoshitomi, S.; Takewaki, Izuru

\section{CITATION:}

Yoshitomi, S....[et al]. Noise-bias compensation in physical-parameter system identification under microtremor input. Engineering Structures 2009, 31(2): 580-590

ISSUE DATE:

2009-02

URL:

http://hdl.handle.net/2433/89631

\section{RIGHT:}

c 2009 Elsevier Ltd All rights reserved.; This is not the published version. Please cite only the published version.; この論文は出版社版でありませ ん。引用の際には出版社版をご確認ご利用ください。 


\title{
Noise-bias Compensation in Physical-parameter System Identification under Microtremor Input
}

\author{
S. Yoshitomi and I. Takewaki ${ }^{*}$ \\ Dept. of Urban \& Environmental Eng., Graduate School of Eng., Kyoto University, \\ Kyotodaigaku-Katsura, Nishikyo, Kyoto 615-8540, Japan
}

\begin{abstract}
A direct method of physical-parameter system identification (SI) is developed in the case of containing noises at both floors above and below a specified story. To investigate the effect of the level of noises on the accuracy of identification, numerical simulations are performed in the frequency domain by generating two stationary random processes with the specified levels of power spectra. When the previous method of physical-parameter SI is applied to the case contaminated by noises at both floors just above and below a specified story, it is difficult to evaluate the true stiffness and damping coefficients depending on the intensity. To overcome this difficulty, a new noise-bias compensation method is proposed which enables one to evaluate the intensity of noises in addition to the identification of story stiffness and damping. This method can be used in the case of small SN (Signal to Noise) ratios such as actual microtremor records. The effectiveness of the proposed method is demonstrated through a numerical example and an experiment using actual microtremor records for a reduced two-story model.
\end{abstract}

Keywords: System identification, Noise effect, Structural health monitoring, Stationary random vibration, Microtremor, Stiffness-damping identification

\footnotetext{
* Corresponding author. Tel.: +81-75-383-3294, Fax: +81-75-383-3297, E-mail: takewaki@archi.kyoto-u.ac.jp
} 


\section{Introduction}

System identification (SI) techniques play important roles in identifying discrepancies between constructed structural systems and the corresponding design models and enhancing the reliability of the design system including such design models. SI techniques have been studied extensively and many useful accomplishments have been accumulated so far (see, for example, [1-20]). Structural health monitoring for damage detection is also indebted to these SI techniques. Several useful methods for damage detection have been proposed [21-24] in which finite-element models are used for identifying the location of damage. To know the structural parameters without damage and under ordinary environments, it is desired to establish the method for SI by using stationary vibration records. In the case of using microtremor records for SI, the SN (Signal to Noise) ratio may be quite small and the unfavorable effects of noises on SI may be significant. There are few researches on the effect of noises on SI and the method for elimination of the effect of noises. In this paper, a new noise-bias compensation method is proposed in which unfavorable effects of noises on SI can be eliminated.

There are two major approaches in SI [1], i.e. the modal-parameter SI and the physicalparameter SI. The former is thought to be appropriate for identifying the overall dynamic properties of a structural system and usually exhibits stable characteristics. While the latter is important from the viewpoint of enhancement of reliability in structural controlled systems [17] and provides direct information on structural parameters, its development is limited because of the requirement of multiple measurements or the necessity of complicated manipulation. A hybrid approach is sometimes used in which physical parameters are identified from the modal data obtained by the modal-parameter SI. However, a sufficient number of modal data must be prepared for the unique and accurate identification of the physical parameters. This is usually difficult to be met.

In general, acceleration records in all the floors above the story with the stiffness and damping to be identified are necessary to evaluate the story shear force required in the stiffness-damping evaluation. Although the cost of sensors is getting cheaper recently, this instrumentation and data management may not be cost-effective in multi-storied buildings, 
especially in high-rise buildings. To avoid this, Udwadia et al. [25] proposed a unique SI theory for a shear building model. They clarified that unique identification of story stiffnesses and viscous damping coefficients is possible theoretically when acceleration records at the floors just above and below a specific story are available. While the applicability of their theory to actual earthquake records with noises is not clear, it provides a possibility of constructing a theoretical basis for the physical-parameter SI.

In the previous papers [26, 27], physical-parameter SI methods have been proposed to identify a story stiffness, a material damping ratio and a viscous damping coefficient in a shear building model. The mathematical limit manipulation toward an infinite frequency [25] has been avoided by introducing a limit manipulation toward zero frequency together with an interpolation technique. In that method, both the material and viscous damping properties together with the story stiffness can be identified simultaneously in a unified manner and only an FFT technique is needed. However the previous methods have a drawback of noisesensitive properties. In the paper [27], the effects of noises have been eliminated partially when a record at either one of floors just above or below a specified story contains a noise. In a realistic situation, however, both records are usually contaminated by noises. Furthermore, in microtremors, the intensity of noise seems to be relatively large compared to the true signal (small SN ratio). In these cases the effect of noises is significant.

In this paper, a new noise-bias compensation method is proposed in which unfavorable effects by noises can be eliminated. This method enables one to evaluate the intensity of noises in addition to the identification of story stiffness and damping. The effectiveness of the proposed method is demonstrated through a numerical example and an experiment using actual microtremor records in a reduced two-story model.

\section{Stiffness-Damping Simultaneous Identification}

\subsection{Identification of stiffness and material damping}

Consider an $\mathrm{N}$-story shear building model, as shown in Fig.1, with viscous and material dampings. The node and element numbers are defined from the top. The $j$ th node and the $j$ th element from the top are called the "node $j$ " and the "element $j$ ". Let $m_{j}$ and $k_{j}$ denote the 
mass of the node $j$ and the story stiffness of the element $j$ and let $c_{j}$ and $\beta_{j}$ be the viscous damping coefficient and the material damping ratio in the element $j$. The complex stiffness of the element $j$ is described as $k_{j}^{+}=k_{j}\left(1+2 \beta_{j} \mathrm{i}\right)$ where i indicates the imaginary unit.

The frequency-domain equations of motion for this shear building model subjected to the horizontal base acceleration $\ddot{z}(t)$ is expressed by

$$
\mathbf{A}(\omega) \mathbf{U}(\omega)=\mathbf{F}(\omega) Z(\omega)
$$

$\mathbf{U}(\omega)$ and $Z(\omega)$ are the Fourier-transforms of the nodal absolute horizontal displacements $\mathbf{u}(t)$ and the base displacement $z(t)$, respectively. The vector $\mathbf{F}(\omega)$ in Eq.(1) indicates $\mathbf{F}(\omega)=\left\{\begin{array}{llll}0 & 0 & \cdots & \mathrm{i} \omega \boldsymbol{C}_{N}+k_{N}{ }^{+}\end{array}\right\}^{T}$ and the symbol ()$^{T}$ means the transpose of a vector. The matrix $\mathbf{A}(\omega)$ in Eq.(1) is defined as

$$
\begin{aligned}
& \mathbf{A}(\omega)=-\omega^{2} \mathbf{M}+\mathrm{i} \omega \mathbf{C}+\mathbf{K}
\end{aligned}
$$

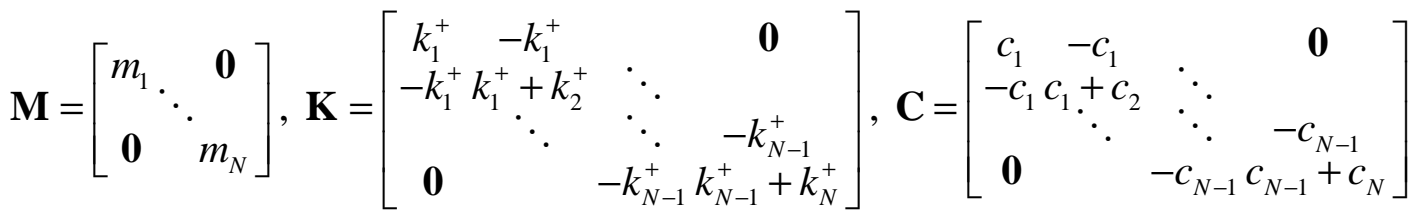

The $j$ th component $U_{j}(\omega)$ in $\mathbf{U}(\omega)$ can be obtained from Eq.(1) as

$$
U_{j}(\omega)=\frac{\Delta_{j}(\omega)}{P_{N}(\omega)} Z(\omega)
$$

$P_{j}(\omega)$ is the determinant of the upper-left $j \times j$ submatrix of $\mathbf{A}(\omega)$ and $\Delta_{j}(\omega)$ is the determinant of the matrix obtained from $\mathbf{A}(\omega)$ by replacing its $j$ th column by $\mathbf{F}(\omega)$. The definition of $\mathbf{F}, \Delta_{j}, P_{j}$ and another definition $b_{j}(\omega)=\mathrm{i} \omega c_{j}+k_{j}^{+}$provide the expression of $\Delta_{j}$.

$$
\Delta_{j}= \begin{cases}b_{N} \cdots b_{j} P_{j-1} & (j \geq 2) \\ b_{N} \cdots b_{1} & (j=1)\end{cases}
$$

It should be noted that the matrix for which $\Delta_{j}$ is defined has a tri-diagonal property only for $j=N$ and $\Delta_{N}$ can be expressed rather compactly. This property is closely related to the fact that the story stiffness of the element $N$ and its material damping ratio can be identified 
uniquely when the acceleration $\ddot{u}_{N}(t)$ of the node $N$ is observed in addition to the base acceleration $\ddot{z}(t)$.

Substitution of Eq.(5) into Eq.(4) provides the following relations.

$$
\frac{U_{U}}{U_{L}}= \begin{cases}U_{N} / Z=b_{N} P_{N-1} / P_{N} & \text { (1st story) } \\ U_{j} / U_{j+1}=b_{j} P_{j-1} / P_{j} & \text { ( } j \text { th story, } 2 \leq j \leq N-1) \\ U_{1} / U_{2}=b_{1} / P_{1}=b_{1} / a_{1} & \text { (topstory) }\end{cases}
$$

where $U_{U}$ and $U_{L}$ indicate the Fourier transforms of displacements at floors just above and below the target story, respectively, and $a_{1}(\omega)$ is defined as $a_{1}(\omega)=-\omega^{2} m_{1}+b_{1}(\omega)$. After some manipulations [26], the following relations can be derived (Eq.(7c) is a new one).

$$
\left\{\begin{array}{cl}
\lim _{\omega \rightarrow 0} \frac{(\mathrm{i} \omega)^{2} \ddot{U}_{N}}{\ddot{Z}-\ddot{U}_{N}}=\frac{k_{N}\left(1+2 \beta_{N} \mathrm{i}\right)}{M_{N}} & \text { (1st story) } \\
\lim _{\omega \rightarrow 0} \frac{(\mathrm{i} \omega)^{2} \ddot{U}_{j}}{\ddot{U}_{j+1}-\ddot{U}_{j}}=\frac{k_{j}\left(1+2 \beta_{j} \mathrm{i}\right)}{M_{j}} & \text { (jth story, } 2 \leq j \leq \mathrm{N}) \\
\frac{(\mathrm{i} \omega)^{2} \ddot{U}_{1}}{\ddot{U}_{2}-\ddot{U}_{1}}=\frac{k_{1}}{m_{1}}+\frac{\omega c_{1}+2 k_{1} \beta_{1}}{m_{1}} \mathrm{i} & \text { (top story) }
\end{array}\right.
$$

where $M_{j}=\sum_{i=1}^{j} m_{i} \cdot \ddot{U}_{j}$ and $\ddot{Z}$ indicate the Fourier transforms of acceleration $\ddot{u}_{j}(t)$ and $\ddot{z}(t)$, respectively. Eqs.(7a, b) show that the stiffness of the target story except the top story can be identified as a limit value of a certain function. On the other hand, the function for stiffness identification of the top story can be expressed as Eq.(7c) and is different from that of the other story. This is because, while $P_{j}$ in Eq.(6) satisfies a recurrence formula [26] and the expression of $P_{j}$ is complex, the real part of the function in Eq.(7c) has a constant value and the stiffness can be identified without limit manipulation. Since these functions, Eqs.7(a, b), have almost the same form, the function for the first story will be dealt with hereafter as a representative one.

The identification of the viscous damping coefficient $c_{N}$ is also possible by introducing the limit manipulation toward zero frequency of the slope of the characteristic function [27].

\subsection{Application to stationary random vibration}

Consider stationary random records hereafter. It is assumed in this section that all the 
records are noise-free. The noise problem will be discussed in Section 3 and 4 .

Let $S_{N N}, S_{Z Z}$ denote the power spectra of $\ddot{u}_{N}(t)$ and $\ddot{z}(t)$, respectively, and $S_{N Z}, S_{Z N}$ denote the cross spectra between $\ddot{u}_{N}(t)$ and $\ddot{z}(t)$ and between $\ddot{z}(t)$ and $\ddot{u}_{N}(t)$, respectively. It can be shown that the following relations hold.

\section{[Property A]}

$$
\lim _{\omega \rightarrow 0} \frac{(\mathrm{i} \omega)^{2} S_{Z N}}{S_{Z Z}-S_{Z N}}=\frac{k_{N}\left(1+2 \beta_{N} \mathrm{i}\right)}{M}
$$

\section{[Property B]}

$$
\lim _{\omega \rightarrow 0} \frac{(\mathrm{i} \omega)^{2} S_{N N}}{S_{N Z}-S_{N N}}=\frac{k_{N}\left(1+2 \beta_{N} \mathrm{i}\right)}{M}
$$

\section{[Property C]}

$$
c_{N}=\lim _{\omega \rightarrow 0}\left(M \times \frac{d}{d \omega}\left\{\operatorname{Im}\left[\frac{(\mathrm{i} \omega)^{2} S_{Z N}}{S_{Z Z}-S_{Z N}}\right]\right\}\right)
$$

or

$$
c_{N}=\lim _{\omega \rightarrow 0}\left(M \times \frac{d}{d \omega}\left\{\operatorname{Im}\left[\frac{(\mathrm{i} \omega)^{2} S_{N N}}{S_{N Z}-S_{N N}}\right]\right\}\right)
$$

\section{[Property AA]}

$$
\begin{aligned}
& k_{N}=\lim _{\omega \rightarrow 0} \operatorname{Re}\left\{M \times(\mathrm{i} \omega)^{2} \times f_{K 1}(\omega)\right\} \\
& \beta_{N}=\lim _{\omega \rightarrow 0} \operatorname{Im}\left\{M \times(\mathrm{i} \omega)^{2} \times f_{K 1}(\omega)\right\} \\
& \text { where } f_{K 1}(\omega)=\frac{S_{Z N}}{S_{Z Z}-S_{Z N}}
\end{aligned}
$$

\section{[Property BB]}

$$
\begin{aligned}
& k_{N}=\lim _{\omega \rightarrow 0} \operatorname{Re}\left\{M \times(\mathrm{i} \omega)^{2} \times f_{K 2}(\omega)\right\} \\
& \beta_{N}=\lim _{\omega \rightarrow 0} \operatorname{Im}\left\{M \times(\mathrm{i} \omega)^{2} \times f_{K 2}(\omega)\right\} \\
& \text { where } f_{K 2}(\omega)=\frac{S_{N N}}{S_{N Z}-S_{N N}}
\end{aligned}
$$

\section{[Property CC]}

$$
c_{N}=\lim _{\omega \rightarrow 0} M \frac{d}{d \omega}\left\{(\mathrm{i} \omega)^{2} \times f_{K 1}(\omega)\right\}
$$




$$
c_{N}=\lim _{\omega \rightarrow 0} M \frac{d}{d \omega}\left\{(\mathrm{i} \omega)^{2} \times f_{K 2}(\omega)\right\}
$$

The proof of these properties can be found in the Ref. [27].

\section{Effect of noise on identification}

\subsection{Formulation under noisy environment}

Assume that the base acceleration $\hat{\ddot{z}}(t)$ consists of the true record $\ddot{z}(t)$ and the corresponding noise $\overline{\bar{w}}_{Z}(t)$.

$$
\hat{\ddot{z}}(t)=\ddot{Z}(t)+\overline{\bar{w}}_{Z}(t)
$$

$\ddot{Z}(t)$ and $\overline{\bar{w}}_{Z}(t)$ are assumed to be stationary random processes. Similarly assume that the upper floor acceleration $\hat{\ddot{u}}_{N}(t)$ consists of the true record $\ddot{u}_{N}(t)$ and the corresponding noise $\overline{\bar{w}}_{N}(t)$ as a stationary random process.

$$
\hat{\ddot{u}}_{N}(t)=\ddot{u}_{N}(t)+\overline{\bar{w}}_{N}(t)
$$

Fig.2(a) shows examples of simulated accelerations at the base and the first floor and Fig.2(b) presents examples of simulated accelerations of noises. The combined simulated accelerations are shown in Fig.2(c). The levels of these signals and noises are determined so as to be comparable to the recorded microtremor accelerations, shown in Fig.2(d), in a 2-story experimental reduced steel frame which will be explained later.

Fourier transformation of Eqs.(18) and (19) leads to

$$
\begin{aligned}
& \hat{\ddot{Z}}=\ddot{Z}+\overline{\bar{W}}_{Z} \\
& \hat{\tilde{U}}_{N}=\ddot{U}_{N}+\overline{\bar{W}}_{N}
\end{aligned}
$$

Let, $S_{\overline{\bar{Z}} Z}, S_{\overline{\bar{Z}} N}, S_{\overline{\bar{N} Z}}, S_{\overline{\bar{N}} N}, S_{\overline{\bar{Z}} \overline{\bar{N}}}$ denote the cross spectra between $\overline{\bar{W}}_{Z}$ and $\ddot{Z}, \overline{\bar{W}}_{Z}$ and $\ddot{U}_{N}, \overline{\bar{W}}_{N}$ and $\ddot{Z}, \overline{\bar{W}}_{N}$ and $\ddot{U}_{N}, \overline{\bar{W}}_{Z}$ and $\overline{\bar{W}}_{N}$, respectively. The power spectra of $\overline{\bar{w}}_{Z}(t)$ and $\overline{\bar{w}}_{N}(t)$ are denoted by $S_{\overline{\bar{Z}} \overline{\bar{Z}}}, S_{\overline{\bar{N}} \overline{\bar{N}}}$, respectively. When the true records and their noises are 
statistically independent and the both noises have no correlation, the following relations hold.

$$
\begin{aligned}
& S_{\overline{\bar{Z} Z}}=0 \\
& S_{N \overline{\bar{N}}}=0 \\
& S_{\overline{\bar{Z}} \overline{\bar{N}}}=0
\end{aligned}
$$

Introducing the transfer function $H(\omega)$ by $\ddot{U}_{N}(\omega)=H(\omega) \ddot{Z}(\omega)$, the following relations hold.

$$
\begin{aligned}
& S_{\overline{\bar{N} Z}}=E\left[\frac{2 \pi}{T} \ddot{Z}^{*} \overline{\bar{W}}_{N}\right]=E\left[\frac{2 \pi}{T} \frac{\ddot{U}_{N}^{*}}{H^{*}(\omega)} \overline{\bar{W}}_{N}\right]=\frac{S_{N \overline{\bar{N}}}}{H^{*}(\omega)}=0 \\
& S_{\overline{\bar{Z} N}}=E\left[\frac{2 \pi}{T} \ddot{U}_{N}^{*} \overline{\bar{W}}_{Z}\right]=E\left[\frac{2 \pi}{T} H^{*}(\omega) \ddot{Z}^{*} \overline{\bar{W}}_{Z}\right]=H^{*}(\omega) S_{Z \overline{\bar{Z}}}=0
\end{aligned}
$$

$T$ indicates the time duration of window and ()$^{*}$ means the complex conjugate of a number. $E[\cdot]$ denotes the ensemble mean.

From Eqs.(22)-(26), the power spectra of $\hat{\ddot{Z}}$ and $\hat{\ddot{U}}_{N}$ and the cross spectrum between $\hat{\ddot{Z}}$ and $\hat{\ddot{U}}_{N}$ are expressed as

$$
\begin{aligned}
& S_{\hat{Z} \hat{Z}}=S_{Z Z}+S_{\overline{\bar{Z} Z}}+S_{Z \overline{\bar{Z}}}+S_{\overline{\overline{Z Z}}}=S_{Z Z}+S_{\overline{\overline{Z Z}}} \\
& S_{\hat{N} \hat{N}}=S_{N N}+S_{N \overline{\bar{N}}}+S_{\overline{\bar{N} N}}+S_{\overline{\bar{N}} \overline{\bar{N}}}=S_{N N}+S_{\overline{\bar{N}} \overline{\bar{N}}} \\
& S_{\hat{Z} \hat{N}}=S_{Z N}+S_{Z \overline{\bar{N}}}+S_{\overline{\bar{Z}} N}+S_{\overline{\bar{Z}} \overline{\bar{N}}}=S_{Z N}
\end{aligned}
$$

To investigate the effect of noise on the accuracy of identification, assume that the base acceleration is white and the power spectral density levels of the noises at both floors are expressed in terms of the coefficients $a^{2}, b^{2}$ multiplied on the reference power spectra $\bar{S}_{A}$ and $\bar{S}_{B}$.

$$
\begin{aligned}
& S_{\overline{\overline{Z Z}}}=a^{2} \bar{S}_{A} \\
& S_{\overline{\bar{N}} \overline{\bar{N}}}=b^{2} \bar{S}_{B}
\end{aligned}
$$


As will be stated in section $4, \bar{S}_{A}$ and $\bar{S}_{B}$ are the functions to express any shape of power spectra of noises and this expression can be applied to the case where the noise is not white. However, since the specification of these functions is difficult and the white noise with a constant value with respect to frequency is the most fundamental one for the expression of noise, the white noise assumption of noises has been employed.

\subsection{Simulation method in frequency domain}

A simulation method is introduced to demonstrate the validity of the proposed method. Since the present formulation is based on the formulation in the frequency domain, the treatment of noises in the frequency domain seems to be direct. The data $\ddot{Z}$ and $\ddot{U}_{N}$ are therefore treated here in the frequency domain. The upper floor acceleration $\ddot{U}_{N}$ corresponding to the given data $\ddot{Z}$ at the base is generated by using the relation $\ddot{U}_{N}(\omega)=H(\omega) \ddot{Z}(\omega)$. The data $\overline{\bar{W}}_{Z}$ and $\overline{\bar{W}}_{N}$ of noise are also generated in the frequency domain. Applicability of the present formulation in the time domain will be investigated in section 4.3 for actual microtremor records.

The data $\ddot{Z}$ with the prescribed power spectral density $S_{Z Z}$ are generated by using the relation.

$$
\ddot{Z}\left(\omega_{k}\right)=\sqrt{\frac{T}{2 \pi} S_{Z Z}}\left(\cos \theta_{Z}\left(\omega_{k}\right)+i \sin \theta_{Z}\left(\omega_{k}\right)\right), k=0, \ldots, n
$$

where $\theta_{Z}\left(\omega_{k}\right)$ defined in the range $(0,2 \pi)$ are a set of uniform random numbers and $\omega_{k}=\omega^{L}+k \Delta \omega, \Delta \omega=\left(\omega^{U}-\omega^{L}\right) / n$.

The $m$-th sample set of both noises $\overline{\bar{W}}_{Z}$ and $\overline{\bar{W}}_{N}$ with the prescribed power spectral density functions $S_{\overline{\overline{Z Z}}}$ and $S_{\overline{\bar{N}} \overline{\bar{N}}}$ is given by

$$
\begin{aligned}
& \overline{\bar{W}}_{Z}^{(m)}\left(\omega_{k}\right)=\sqrt{\frac{T}{2 \pi} S_{\overline{\bar{Z}}}\left(\omega_{k}\right)}\left(\cos \theta_{k}^{(m)}+\mathrm{i} \sin \theta_{k}^{(m)}\right) k=0, \ldots, n \\
& \overline{\bar{W}}_{N}^{(m)}\left(\omega_{k}\right)=\sqrt{\frac{T}{2 \pi} S_{\overline{\bar{N}} \overline{\bar{N}}}\left(\omega_{k}\right)}\left(\cos \gamma_{k}^{(m)}+\mathrm{i} \sin \gamma_{k}^{(m)}\right) k=0, \ldots, n
\end{aligned}
$$

where the phase angles $\theta_{k}^{(m)}, \gamma_{k}^{(m)}$ defined in the range $(0,2 \pi)$ are uniform random numbers 
independent of $m, k$.

In this paper numerical simulations are performed as follows. First, with the help of Eqs.(32)-(34), Fourier spectra $\ddot{Z}, \ddot{U}_{N}, \overline{\bar{W}}_{Z}$ and $\overline{\bar{W}}_{N}$ are generated. Second, with the help of Eqs.(20),(21), Fourier spectra $\hat{Z}, \hat{\tilde{U}}_{N}$ are generated. Finally, power and cross spectra $S_{\hat{Z} \hat{Z}}, S_{\hat{N} \hat{N}}, S_{\hat{N} \hat{Z}}$ including noises are calculated from $\hat{\ddot{Z}}, \hat{\ddot{U}}_{N}$. By substituting these spectra into Eqs.(11)-(17b), identification in the case with noises is simulated. In a realistic case, $\hat{\ddot{Z}}, \hat{\ddot{U}}_{N}$ are usually calculated by performing Fourier transformation of recorded data $\hat{z}, \hat{\ddot{u}}_{N}$ containing noises. This will be shown in section 4.3.

\subsection{Numerical examination}

A two-story shear building model is considered. Each floor mass is $1 \mathrm{~kg}$. Each story stiffness is $1000 \mathrm{~N} / \mathrm{m}$ and the stiffness-proportional viscous damping is assumed. The lowestmode damping ratio is 0.02 .

As stated in section 3.1, the base acceleration is assumed to be white. The power spectral density function of the base acceleration is specified as $S_{Z Z}=1.0\left(\mathrm{~m}^{2} / \mathrm{s}^{3}\right)$ and $\bar{S}_{A}=\bar{S}_{B}=1.0\left(\mathrm{~m}^{2} / \mathrm{s}^{3}\right)$ are given. The Fourier amplitude spectrum of the base acceleration is generated based on these data and the Fourier amplitude spectrum of the upper floor acceleration is generated by using the transfer function. It should be noted that, while the transfer function is not known actually in the identification procedure, the objective of this section is to demonstrate the effect of noises on the accuracy of identification. Therefore the transfer function is used to generate the power spectrum of the acceleration data at the upper floor. The Fourier amplitude spectra of noises are generated by using several parameters of the power spectral density functions $S_{\overline{\bar{Z}} \overline{\bar{Z}}}=S_{\overline{\bar{N}} \overline{\bar{N}}}=0.1,0.2,0.3,0.4,0.5\left(\mathrm{~m}^{2} / \mathrm{s}^{3}\right)$, i.e. $a^{2}=b^{2}=0.1$, $0.2,0.3,0.4,0.5$. The noise level corresponding to $a^{2}=b^{2}=0.1$ is called ' $10 \%$ noise' hereafter. The power spectral density functions of the base acceleration and the upper floor acceleration are evaluated from the ensemble mean of 300 samples.

Fig.3 shows the effect of the level of noises at both floors on the accuracy of identification. The level of noise has been specified as $a^{2}=b^{2}=0.1,0.2,0.3,0.4,0.5(10-50 \%$ noises). Figs.3(a) and (b) present the real part of the characteristic functions for SI (Eqs.(11) 
and (14)). Figs.3(c) and (d) show the imaginary part of the same functions to identify damping coefficients. From Figs.3(a-d), the following facts are observed.

(1)When the data do not include noises, the limit value converges to the exact stiffness, and the two functions of property AA and BB coincide with each other (Figs.3(a) and (b)).

(2) When the data at both floors contain noises, the shape of characteristic functions for SI is different from that of the case without noise, even if the level of noise is small (Figs.3(a) and (b)).

(3) Around the fundamental natural frequency $(3.11 \mathrm{~Hz})$, the effect of noises is very small and the characteristic functions have large power. As a result, the effect of noises becomes relatively small.

(4)It can be said from Figs.3(c) and (d) that the observation as obtained in Figs.3(a) and (b) is also true in the case of the identification of damping coefficients.

Through the examinations, it is found that the effect of noises on SI in the case of identification of stiffness is not negligible depending on the level of noises. Therefore it is strongly desired to introduce a reliable noise-bias compensation method.

\section{Noise-bias elimination method}

\subsection{Method for estimation of noise level and compensation of noise effect}

A method is proposed here for the evaluation of noise levels in the case where the SN ratio is relatively small. The method proposed in this section uses an advantageous property, as shown in section 3, that two functions $\operatorname{Re}\left[M(\mathrm{i} \omega)^{2} \hat{f}_{K 1}(\omega)\right]$ and $\operatorname{Re}\left[M(\mathrm{i} \omega)^{2} \hat{f}_{K 2}(\omega)\right]$ for stiffness identification do not coincide with each other in the case of including noises at both floors, but coincide with each other in the case without noises. This method uses compensated spectra. If the compensated spectra do not contain noises, above-mentioned two functions for stiffness identification calculated from the compensated spectra coincide with each other. Therefore, this method can determine noise levels so as to minimize the error between two functions $\operatorname{Re}\left[M(\mathrm{i} \omega)^{2} \hat{f}_{K 1}(\omega)\right]$ and $\operatorname{Re}\left[M(\mathrm{i} \omega)^{2} \hat{f}_{K 2}(\omega)\right]$.

When the estimated noise levels are indicated by $a^{\prime 2}$ and $b^{\prime 2}$, the power spectra $S_{\hat{Z} \hat{Z}}$ and $S_{\hat{N} \hat{N}}$ including noises can be compensated as follows by using Eqs.(27)-(31). 


$$
\begin{aligned}
& S_{Z Z}^{\prime}=S_{\hat{Z} \hat{Z}}-a^{\prime 2} \bar{S}_{A} \\
& S_{N N}^{\prime}=S_{\hat{N} \hat{N}}-b^{\prime 2} \bar{S}_{B}
\end{aligned}
$$

where $S_{Z Z}^{\prime}$ and $S_{N N}^{\prime}$ are the compensated power spectra. In section 3, noises are treated as white and the reference power spectra $\bar{S}_{A}, \bar{S}_{B}$ are constant. Two functions $\operatorname{Re}\left[M(\mathrm{i} \omega)^{2} f^{\prime}{ }_{K 1}(\omega)\right]$ and $\operatorname{Re}\left[M(\mathrm{i} \omega)^{2} f^{\prime}{ }_{K 2}(\omega)\right]$ for stiffness identification calculated from the compensated power spectra are obtained by substituting Eqs.(35) and (36) into Eqs.(11) and (14) instead of $S_{Z Z}$ and $S_{N N}$. Here, $f^{\prime}{ }_{K 1}(\omega)$ and $f^{\prime}{ }_{K 2}(\omega)$ are defined as

$$
\begin{gathered}
f^{\prime}{ }_{K 1}(\omega)=\frac{S_{Z N}}{S^{\prime}{ }_{Z Z}-S_{Z N}}=\frac{S_{\hat{Z} \hat{N}}}{\left(S_{\hat{Z} \hat{Z}}-a^{\prime 2} \bar{S}_{A}\right)-S_{\hat{Z} \hat{N}}} \\
f^{\prime}{ }_{K 2}(\omega)=\frac{S^{\prime}{ }_{N N}}{S_{Z N}-S^{\prime}{ }_{N N}}=\frac{S_{\hat{N} \hat{N}}-b^{2} \bar{S}_{B}}{S_{\hat{Z} \hat{N}}-\left(S_{\hat{N} \hat{N}}-b^{2} \bar{S}_{B}\right)}
\end{gathered}
$$

It is demonstrated theoretically here that two functions $\operatorname{Re}\left[M(\mathrm{i} \omega)^{2} f^{\prime}{ }_{K 1}(\omega)\right]$ and $\operatorname{Re}\left[M(\mathrm{i} \omega)^{2} f^{\prime}{ }_{K 2}(\omega)\right]$ for stiffness identification coincide with each other only in the case without noises. In the case of including noises in data at both floors, the functions $\operatorname{Re}\left[M(\mathrm{i} \omega)^{2} f^{\prime}{ }_{K 1}(\omega)\right]$ and $\operatorname{Re}\left[M(\mathrm{i} \omega)^{2} f^{\prime}{ }_{K 2}(\omega)\right]$ are expressed as follows.

$$
\begin{aligned}
\operatorname{Re}\left[M(\mathrm{i} \omega)^{2} f^{\prime}{ }_{K 1}(\omega)\right] & =\operatorname{Re}\left[M(\mathrm{i} \omega)^{2} \frac{S_{Z N}}{S^{\prime}{ }_{Z Z}-S_{Z N}}\right] \\
& =\operatorname{Re}\left[M(\mathrm{i} \omega)^{2} \frac{S_{Z Z} H(\omega)}{S_{Z Z}-\left(\bar{a}^{2}-a^{\prime 2}\right) \bar{S}_{A}-H(\omega) S_{Z Z}}\right] \\
\operatorname{Re}\left[M(\mathrm{i} \omega)^{2} f^{\prime}{ }_{K 2}(\omega)\right] & =\operatorname{Re}\left[M(\mathrm{i} \omega)^{2} \frac{S^{\prime}{ }_{N N}}{S_{N Z}-S^{\prime}{ }_{N N}}\right] \\
& =\operatorname{Re}\left[M(\mathrm{i} \omega)^{2} \frac{\left\{|H(\omega)|^{2} S_{Z Z}+\left(\bar{b}^{2}-b^{\prime 2}\right) \bar{S}_{B}\right\}}{H(\omega) S_{Z Z}-\left\{|H(\omega)|^{2} S_{Z Z}+\left(\bar{b}^{2}-b^{\prime 2}\right) \bar{S}_{B}\right\}}\right]
\end{aligned}
$$

where $\bar{a}^{2}, \bar{b}^{2}$ are the true noise levels. If the estimated noise levels could be equal to the true levels, i.e $a^{\prime 2}=\bar{a}^{2}, b^{\prime 2}=\bar{b}^{2}$, Eqs.(39) and (40) coincide at every $\omega$ as follows. 


$$
\operatorname{Re}\left[M(\mathrm{i} \omega)^{2} f^{\prime}{ }_{K 1}(\omega)\right]=\operatorname{Re}\left[M(\mathrm{i} \omega)^{2} f^{\prime}{ }_{K 2}(\omega)\right]=M(\mathrm{i} \omega)^{2} \frac{H_{R}(\omega)-|H(\omega)|^{2}}{1+|H(\omega)|^{2}-2 H_{R}(\omega)}
$$

where, $H_{R}(\omega) \equiv \operatorname{Re}[H(\omega)]$. As for the other combination of $a^{\prime 2}$ and $b^{\prime 2}$, Eqs.(39) and (40) do not coincide at every $\omega$, because $H(\omega)$ is not constant. Therefore it is restricted to the case $a^{\prime 2}=\bar{a}^{2}, b^{\prime 2}=\bar{b}^{2}$ that the functions (39) and (40) coincide at every $\omega$. In other word, by finding $\bar{a}^{2}$ and $\bar{b}^{2}$ so as to satisfy the relation $\operatorname{Re}\left[M(\mathrm{i} \omega)^{2} f^{\prime}{ }_{K 1}(\omega)\right]=$ $\operatorname{Re}\left[M(\mathrm{i} \omega)^{2} f^{\prime}{ }_{K 2}(\omega)\right]$, the exact noise level can be estimated.

In the case of using actual records, the assumption that the noises included in records are white does not hold strictly, and the relation $\operatorname{Re}\left[M(\mathrm{i} \omega)^{2} f^{\prime}{ }_{K 1}(\omega)\right]=\operatorname{Re}\left[M(\mathrm{i} \omega)^{2} f^{\prime}{ }_{K 2}(\omega)\right]$ does not hold strictly. Then the following function $F\left(a^{\prime 2}, b^{\prime 2}\right)$ will be introduced to estimate the error between $\operatorname{Re}\left[M(\mathrm{i} \omega)^{2} f^{\prime}{ }_{K 1}(\omega)\right]$ and $\operatorname{Re}\left[M(\mathrm{i} \omega)^{2} f^{\prime}{ }_{K 2}(\omega)\right]$.

$$
F\left(a^{\prime 2}, b^{\prime 2}\right)=\sum_{k=k}^{k^{U}}\left(\operatorname{Re}\left[M(\mathrm{i} \omega)^{2} f^{\prime}{ }_{K 1}\left(\omega_{k}\right)\right]-\operatorname{Re}\left[M(\mathrm{i} \omega)^{2} f^{\prime}{ }_{K 2}\left(\omega_{k}\right)\right]\right)^{2}
$$

where, $k^{L}$ and $k^{U}$ represent the number corresponding to the lower and upper bound, respectively, of the specified frequency range. After some investigations using simulated data, it was found that the number of frequencies, defined by the index $k$, does not affect much the optimization result in the ordinary number of frequency points. However, in case of using actual data, it is recommended to use the minimum increment of frequency which is determined from the number of time-series data and sampling ratio.

This method is devised so that the error between two compensated functions is minimized in the specified frequency range (Fig.4). When the function (42) is minimized with respect to $a^{\prime 2}$ and $b^{\prime 2}$, the functions $\operatorname{Re}\left[M(\mathrm{i} \omega)^{2} f^{\prime}{ }_{K 1}(\omega)\right]$ and $\operatorname{Re}\left[M(\mathrm{i} \omega)^{2} f^{\prime}{ }_{K 2}(\omega)\right]$ can be regarded to coincide with each other, and noise levels $a^{\prime 2}, b^{\prime 2}$ are obtained. By substituting the obtained noise levels $a^{\prime 2}, b^{\prime 2}$ into Eqs. (35) and (36), the functions for SI without the effect of noises can be obtained. Fig.5 shows the flow chart of this method. This method has two advantages in terms of practical application. First, it enables one to estimate noise levels in addition to the system identification (stiffness and damping) in the case of 
using microtremor records with small SN ratios. Second, it retains robustness against unreliable data by excluding a small or large unreliable frequency range in actual microtremor records.

\subsection{Numerical example using the proposed noise-bias compensation method}

The discussion in section 4.1 is verified through a numerical example. A two-story shear building model is considered again. Each floor mass is $1 \mathrm{~kg}$ and each story stiffness is $1000 \mathrm{~N} / \mathrm{m}$. The lowest-mode damping ratio is 0.02 and the power spectral density function of the base acceleration is $S_{Z Z}=1.0\left(\mathrm{~m}^{2} / \mathrm{s}^{3}\right)$. The Fourier amplitude spectrum of the base acceleration is generated based on these data and the Fourier amplitude spectrum of the upper floor acceleration is generated by using the transfer function. The Fourier amplitude spectra of noises are generated by using the level coefficients $a^{2}=0.4, b^{2}=0.6$. As a result, $S_{\overline{\bar{Z}} \overline{\bar{Z}}}=a^{2} \bar{S}_{A}=0.4\left(\mathrm{~m}^{2} / \mathrm{s}^{3}\right)$ and $S_{\overline{\bar{N}} \overline{\bar{N}}}=b^{2} \bar{S}_{B}=0.6\left(\mathrm{~m}^{2} / \mathrm{s}^{3}\right)$ are given. A numerical example is presented here by using $\bar{S}_{A}=\bar{S}_{B}=1.0\left(\mathrm{~m}^{2} / \mathrm{s}^{3}\right)$ for the identification of the first story.

As for the case of using data before compensation, Figs.6(a)-(c) show the relations among the noise level, two functions for stiffness identification and the error (difference) of these functions. Here, the noise levels $a^{2}$ and $b^{2}$ have values in the range from 0.0 to 1.0. The function $\operatorname{Re}\left[M(\mathrm{i} \omega)^{2} \hat{f}_{K 1}(\omega)\right]$ is affected by $S_{\overline{\bar{Z}} \overline{\bar{Z}}}=a^{2} \bar{S}_{A}$ only judging from Eq.(39) and the function $\operatorname{Re}\left[M(\mathrm{i} \omega)^{2} \hat{f}_{K 2}(\omega)\right]$ is affected by $S_{\overline{\bar{N}} \overline{\bar{N}}}=b^{2} \bar{S}_{B}$ only judging from Eq.(40). Fig.6(a) illustrates the effect of the noise level $a^{2}$ on the shape of $\operatorname{Re}\left[M(\mathrm{i} \omega)^{2} \hat{f}_{K 1}(\omega)\right]$ and Fig.6(b) shows the effect of the noise level $b^{2}$ on the shape of $\operatorname{Re}\left[M(\mathrm{i} \omega)^{2} \hat{f}_{K_{2}}(\omega)\right]$. Fig.6(c) indicates the function $\hat{F}\left(a^{2}, b^{2}\right)$ as the error (difference) between $\operatorname{Re}\left[M(\mathrm{i} \omega)^{2} \hat{f}_{K 1}(\omega)\right]$ and $\operatorname{Re}\left[M(\mathrm{i} \omega)^{2} \hat{f}_{K_{2}}(\omega)\right]$. From Figs.6(a)-(c), the following facts are obtained.

(1)The shapes of $\operatorname{Re}\left[M(\mathrm{i} \omega)^{2} \hat{f}_{K 1}(\omega)\right]$ and $\operatorname{Re}\left[M(\mathrm{i} \omega)^{2} \hat{f}_{K 2}(\omega)\right]$ coincide with each other only in the case of $a^{2}=0$ and $b^{2}=0$.

(2) The function $\hat{F}\left(a^{2}, b^{2}\right)$ has the smallest value in the case of $a^{2}=0$ and $b^{2}=0$.

(3) The function $\hat{F}\left(a^{2}, b^{2}\right)$ is a multi-peak function.

On the other hand, as for the case of using data after compensation, Figs.7(a)-(d) show the relations among the noise level, two functions for stiffness identification and the error 
(difference) of these functions. A numerical example is presented here by using $\bar{S}_{A}=\bar{S}_{B}=1.0\left(\mathrm{~m}^{2} / \mathrm{s}^{3}\right), a^{2}=0.20$ and $b^{2}=0.40$ for the same model as stated above. Fig.7(a) illustrates the effect of compensation under an assumed noise level $a^{\prime 2}$ on the stiffness identification function $\operatorname{Re}\left[M(\mathrm{i} \omega)^{2} f^{\prime}{ }_{K 1}(\omega)\right]$, and Fig.7(b) shows the effect of compensation under an assumed noise level $b^{\prime 2}$ on the stiffness identification function $\operatorname{Re}\left[M(\mathrm{i} \omega)^{2} f^{{ }^{\prime}}{ }_{K 2}(\omega)\right]$. The bold lines in Figs.7(a) and (b) are on the vertical plane perpendicular to the axis of $a^{2}$ and through the exact point of noise level. Fig.7(c) shows the function $F\left(a^{\prime 2}, b^{\prime 2}\right)$ as the error (difference) between $\operatorname{Re}\left[M(\mathrm{i} \omega)^{2} f^{\prime}{ }_{K 1}(\omega)\right]$ and $\operatorname{Re}\left[M(\mathrm{i} \omega)^{2} f^{\prime}{ }_{K 2}(\omega)\right]$. Furthermore, the bold lines in Fig.7(c) indicates the optimization process starting from different initial values so as to minimize $F\left(a^{\prime 2}, b^{\prime 2}\right)$ in the frequency range $0 \sim 8 \mathrm{~Hz}$. Here, the gradient projection method is used as an optimization algorithm. Fig.7(d) shows the projection of Fig.7(c) to the $a^{\prime 2}-b^{\prime 2}$ plane. From Figs.7(a)-(d), the following facts are obtained.

(1) The shapes of $\operatorname{Re}\left[M(\mathrm{i} \omega)^{2} f{ }^{\prime}{ }_{K 1}(\omega)\right]$ and $\operatorname{Re}\left[M(\mathrm{i} \omega)^{2} f^{\prime}{ }_{K 2}(\omega)\right]$ coincide with each other only in the case when the compensation noise level is exact $\left(a^{\prime 2}=0.2, b^{\prime 2}=0.4\right)$.

(2) The function $F\left(a^{\prime 2}, b^{\prime 2}\right)$ has the smallest value at $a^{\prime 2}=0.2$ and $b^{\prime 2}=0.4$.

(3) The function $F\left(a^{\prime 2}, b^{\prime 2}\right)$ is a multi-peak function. Therefore, the result of optimization depends on the initial values.

The starting point affects clearly the optimization result caused by multi-peak objective function and there is no definite method for overcoming this. In this paper, an approximate method of starting optimization from different initial values including a solution by the line search method is introduced. In a realistic case, it may be sufficient to obtain an approximate optimum solution in order to reduce noise effects on SI. Furthermore, if an exact optimum solution is desired, an enumeration method can be applied because the present optimization problem includes only two parameters $a^{\prime 2}, b^{\prime 2}$ independent of the number of stories of the model and the computational task is not so heavy.

For some initial values, the noise levels $a^{\prime 2}$ and $b^{\prime 2}$ are obtained as almost exact $\left(a^{\prime 2}=0.21, b^{\prime 2}=0.40\right)$. Fig.8 shows the result of compensation by using the obtained noise level. It is found that the shapes of two functions are not similar before compensation but 
coincide with each other after compensation. It indicates that the proposed method works effectively in this case.

\subsection{Example using actual microtremor records}

The SI of a two-story shear building model as shown in Fig.9 is performed by using actual microtremor records. This model is set on the room floor and the microtremor velocities are recorded through sensors set on the model floor levels. Since the proposed method can be applied also to the velocities, the velocities with high intensity are recorded in this example. The sampling frequency of recording is $250 \mathrm{~Hz}$ and the recording time duration is about 130s. One sample set contains 2048 data in the time domain and Fourier transformation is performed for those data. The power and cross spectra of the recorded data are estimated as ensemble means of 2000 samples generated by shifting the time window by $20 \Delta t$ ( $\Delta t$ : data interval). The lowest floor is called the 1F-floor and the highest floor is called the 3F-floor.

To verify the validity of proposed method, the stiffness and damping of the model are estimated by the modal parameter SI requiring the natural frequencies as many as the number of stories. In the model of two stories, the measurement of two natural frequencies does not seem to be difficult. On the other hand, the measurement of higher natural frequencies of the model with a large number of stories may be difficult and it does not appear to be realistic to apply this modal-parameter SI to the model with a lot of stories. For this reason, the model of two stories has been employed. From the power and cross spectra of the records at 1F-floor and 2F-floor, the fundamental natural frequency is estimated as $3.91 \mathrm{~Hz}$ and the 2nd as 10.38Hz. The masses are $1.48 \mathrm{~kg}$ (2F-floor, containing sensor) and $1.14 \mathrm{~kg}$ (3F-floor). From these values, the stiffnesses of both stories are estimated as $1869 \mathrm{~N} / \mathrm{m}$ (1st story) and 2316N/m (2nd story), respectively. It is assumed here that the stiffness obtained from the modalparameter SI is correct. As for the damping ratio, the lowest-mode damping ratio is estimated as 0.015 by employing the logarithmic decrement method for the record of free vibration. By assuming proportional damping, damping coefficients can be estimated as $2.28 \mathrm{Ns} / \mathrm{m}$ (1st story) and 2.83Ns/m (2nd story). 
The proposed noise-bias compensation method is applied to the microtremor data recorded at the $1 \mathrm{~F}$-floor and the 2F-floor. The functions for SI before compensation are shown in Fig.10. For comparison, figures are shown of simulation including noises at both floors without correlation and with levels estimated in this section. From Figs.10(a), (b), it can be observed that the shapes of the functions using actual records correspond to the shapes of the functions in the simulation before compensation.

To estimate the noise levels, the minimization of the function $F\left(a^{\prime 2}, b^{\prime 2}\right)$ is performed. As stated below, the functions for SI are very sensitive to the noise in the low frequency range from 0 to $3 \mathrm{~Hz}$. Therefore, the frequency range to define $F\left(a^{\prime 2}, b^{\prime 2}\right)$ is taken from 4 to $7 \mathrm{~Hz}$. Fig.11 shows the function $F\left(a^{\prime 2}, b^{\prime 2}\right)$ and the result of optimization from different initial values. As stated in section 4.2, the result of optimization depends on initial values. Therefore, the optimization is performed here from the solution found by using the line search method under the constraint $a^{\prime^{2}}=b^{\prime^{2}}$.

In Figs.11(a) and (b), the blue broken line and the blue circle indicate the direction of line search and its minimum solution, respectively. A curved surface is drawn by changing $a^{\prime 2}$ and $b^{\prime 2}$ independently from 0.0 to 1.0 and the red circle indicates the minimum point of the curved surface. Here, the red circle is regarded as the global minimum solution, i.e. the exact solution. From Figs.11(a) and (b), it can be observed that the obtained optimum solution starting from the blue circle does not coincide with the exact solution. Therefore, the solution is adopted which has the minimum objective function among the solutions obtained by starting from different initial values. It should be noted that, in case of using actual records, the assumption that noises are white does not hold exactly, and the error function, Eq.(42), does not approach to zero exactly. Therefore, in case of using actual records, it is not necessary to obtain the global minimum solution and it is sufficient to obtain the solution having a smaller value of the objective function than that before compensation in order to reduce the effect of noises. It may also be possible, if desired, to obtain the global optimum solution by changing $a^{\prime 2}$ and $b^{\prime 2}$ independently because this problem of decreasing the error function has only two parameters $a^{\prime^{2}}$ and $b^{\prime^{2}}$.

The noise levels have been estimated as $a^{\prime^{2}}=0.66$ and $b^{\prime^{2}}=0.55$ in case of setting 
$\bar{S}_{A}=\bar{S}_{B}=0.43 \times 10^{-4}\left(\mathrm{~m}^{2} / \mathrm{s}\right)$. This value $0.43 \times 10^{-4}\left(\mathrm{~m}^{2} / \mathrm{s}\right)$ comes from the average value of the power spectrum of an actual record at the base. These noise levels correspond to 1.97 and 1.63, respectively, if they are estimated as ratios to $S_{Z Z}$ without noise. The results of compensation using these noise levels are shown in Fig.12. Fig.12 indicates that, while the shapes of functions of Property AA and BB do not coincide with each other before compensation, the shapes of these functions almost coincide with each other after compensation. As shown in Fig.12, these functions are very sensitive to the noise and the effect of compensation is not enough in a low frequency range from 0 to $3 \mathrm{~Hz}$. To evaluate the stiffness as a limit value of these functions, the extrapolation is introduced here by using an approximate function $k(f)=A\left(f^{3}-f_{1}^{3}\right)+\bar{k}$. This function is set based on the characteristic that the value at the fundamental natural frequency $\left(f_{1}=3.91 \mathrm{~Hz}\right.$ ) is affected slightly by noises. The coefficient $A$ is obtained so as to minimize the sum of the squared errors from the approximate function in a range of frequency from $4 \mathrm{~Hz}$ to $7 \mathrm{~Hz}$. As a result of application of this process to the function of property AA, the stiffness is estimated as $1801 \mathrm{~N} / \mathrm{m}$ at the limit $\omega \rightarrow 0$ and this value is almost the same as the exact value $(1869 \mathrm{~N} / \mathrm{m})$ obtained by the aforementioned modal-parameter SI.

Figs.12(c) and (d) show that the function for identification of the viscous damping coefficient is very sensitive to the noises. In this case, the difficulty in damping coefficient identification may be caused by a very low level of damping. This problem needs further discussion.

\section{Conclusions}

A physical-parameter SI method was proposed in the previous paper [27]. In that paper, the effects of extraneous noises can be eliminated when a record at either one of floors just above or below a specified story contains a noise. In a realistic situation, however, both of records usually contain noises. Furthermore, in the records of microtremors as a representative of stationary records, the level of noise seems to be relatively large compared to the true signal. In these cases the effect of noises on the accuracy of SI is not negligible.

A more reliable and stable method of physical-parameter SI under noisy environments 
has been proposed in this paper compared to the previous one based on the power and cross spectra. The advantageous features of this method may be summarized as follows:

(1) In the previous papers [26, 27], the examination of the effect of the level and correlation of noises on the SI was not performed sufficiently. Through numerical examples in the frequency domain, the effect of noise level on the accuracy of identification has been investigated in this paper.

(2) When the previous system identification method [27] is applied to the case including noises at both floors just above and below a specified story, the shape of characteristic functions to evaluate the stiffness and damping coefficients is different greatly from that of the case without noise. In the case where the level of noises is large, it is difficult to evaluate the true stiffness and damping coefficients even though an extrapolation technique is used. Therefore it is necessary to introduce a new noise compensation method.

(3) A new noise-bias elimination methods can be developed. This method has an advantage that it does not require the limit value in the sensitive frequency regions. Therefore, this method can be applied in the case of using actual data with a small $\mathrm{SN}$ ratio such as microtremor records. This method can capture the noise levels by minimizing the error between two functions for SI corresponding to Property AA and BB in which the compensated power and cross spectra are substituted. This process is based on the characteristics that in the case without noises, two functions for SI corresponding to Property AA and BB coincide with each other.

(4) Numerical examples demonstrated that the proposed method works very well in both cases of using simulated data and actual recorded data. It has been shown that the characteristic functions for SI can be obtained in a stable way even under noisy environments.

\section{Acknowledgements}

This work is partially supported by the Grant-in-Aid for Scientific Research (No.18656156) from the Ministry of Education, Science, Sports and Culture of Japan. The 
authors are also grateful to Dr. M.Nakamura of Obayashi Co. for providing us with useful comments.

\section{References}

[1] Hart GC, Yao JTP. System identification in structural dynamics. J. Engng. Mech. Div., ASCE 1977; 103(EM6): 1089-1104.

[2] Beck JL, Jennings, PC. Structural identification using linear models and earthquake records. Earthquake Engng. Struct. Dyn. 1980; 8: 145-160.

[3] Hoshiya M, Saito E. Structural identification by extended Kalman filter. J. Engng. Mech., ASCE 1984; 110(12): 1757-1770.

[4] Safak E. Adaptive modeling, identification, and control of dynamic structural systems I: Theory. J. Engng. Mech., ASCE 1989; 115(11): 2386-2405.

[5] Agbabian MS, Masri SF, Miller RK, Caughey TK. System identification approach to detection of structural changes. J. Engng. Mech., ASCE 1991; 117(2): 370-390.

[6] Koh CG., See LM, Balendra T. Estimation of structural parameters in time domain: a substructure approach. Earthquake Engng. Struct. Dyn. 1991; 20: 787-801.

[7] Hong K-S., Yun C.-B., Improved method for frequency domain identifications of structures, Engineering Structures 1993; 15(3): 179-188.

[8] Yao JTP, Natke HG. Damage detection and reliability evaluation of existing structures. Structural Safety 1994; 15: 3-16.

[9] Ghanem R, Shinozuka M. Structural-system identification I: Theory. J. Engng. Mech., ASCE 1995; 121(2): 255-264.

[10] Hjelmstad KD, Banan MoR, Banan MaR. On building finite element models of structures from modal response. Earthquake Engng. Struct. Dyn. 1995; 24: 53-67.

[11] Shinozuka M, Ghanem R. Structural-system identification II: Experimental verification. J. Engng. Mech., ASCE 1995; 121(2): 265-273.

[12] Koh CG., See LM., Balendra T. Determination of storey stiffness of three-dimensional frame buildings, Engineering Structures 1995; 17(3): 179-186.

[13] Doebling SW, Farrar CR, Prime MB, Shevitz DW. Damage identification and health 
monitoring of structural and mechanical systems from changes in their vibration characteristics: A literature review. Los Alamos National Laboratory Report LA-13070MS, 1996.

[14] Hjelmstad KD. On the uniqueness of modal parameter estimation. J. Sound and Vib. 1996; 192(2): 581-598.

[15] Masri SF, Nakamura M, Chassiakos AG, Caughey TK. A neural network approach to the detection of changes in structural parameters. J. Engng. Mech., ASCE 1996; 122(4): 350360.

[16] Tan RY., Weng IW. Identification of dynamic properties of isolated structures, Engineering Structures 1996; 18(3): 240-246.

[17] Housner G., et al. Special issue, Structural control: past, present, and future. J. Engng. Mech., ASCE 1997; 123(9): 897-971.

[18] Herrmann T, Pradlwarter HJ. Two-step identification approach for damped finite element models. J. Engng. Mech., ASCE 1998; 124(6): 639-647.

[19] Liu H., Yang Z., Gaulke MS. Structural identification and finite element modeling of a 14-story office building using recorded data, Engineering Structures 2005; 27(3):463473.

[20] Fourth World Conference on Structural Control and Monitoring (4WCSCM), San Diego, 11-13, July, 2006.

[21] Jaishi B, Ren WX. Damage detection by finite element model updating using modal flexibility residual, J. Sound and Vib., 2006; 290, 369-387.

[22] Perera R, Ruiz A. A multistage FE updating procedure for damage identification in largescale structures based on multiobjective evolutionary optimization, Mechanical Systems and Signal Processing, 2008; 22(4), 970-991.

[23] Friswell MI. Damage identification using inverse methods, Philosophical Transactions of the Royal Society, 2007; 365(1851), 393-410.

[24] Wang J-F., Lin C-C., Yen S-M. A story damage index of seismically-excited buildings based on modal frequency and mode shape, Engineering Structures 2007; 29(9): 21432157. 
[25] Udwadia FE, Sharma DK, Shah PC. Uniqueness of damping and stiffness distributions in the identification of soil and structural systems. J. Appl. Mech., ASME 1978; 45: 181187.

[26] Takewaki I, Nakamura M. Stiffness-damping simultaneous identification using limited earthquake records. Earthquake Engng. Struct. Dyn. 2000; 29(8): 1219-1238.

[27] Takewaki I, Nakamura M. Stiffness-damping simultaneous identification under limited observation. J. Engng. Mech., ASCE 2005; 131(10): 1027-1035. 
Fig.1 Shear building model and measurement at floors just below and above the target story Fig.2 Simulated and recorded acceleration

Fig.3 Effect of noise level on accuracy of identification

Fig.4 Concept of the proposed noise-bias compensation method

Fig.5 Flow chart of proposed noise-bias compensation method

Fig.6 Effect of noise level on error function before compensation

Fig.7 Effect of noise level on error function after compensation and optimization process

Fig.8 Effect of noise-bias compensation on accuracy of identification

Fig.9 2-story experimental steel frame

Fig.10 Stiffness and damping identification of first story

Fig.11 Optimization process from various starting points and constrained line $a^{\prime^{2}}=b^{\prime^{2}}$ used for line search

Fig.12 Effect of noise-bias compensation method (first story identification) 


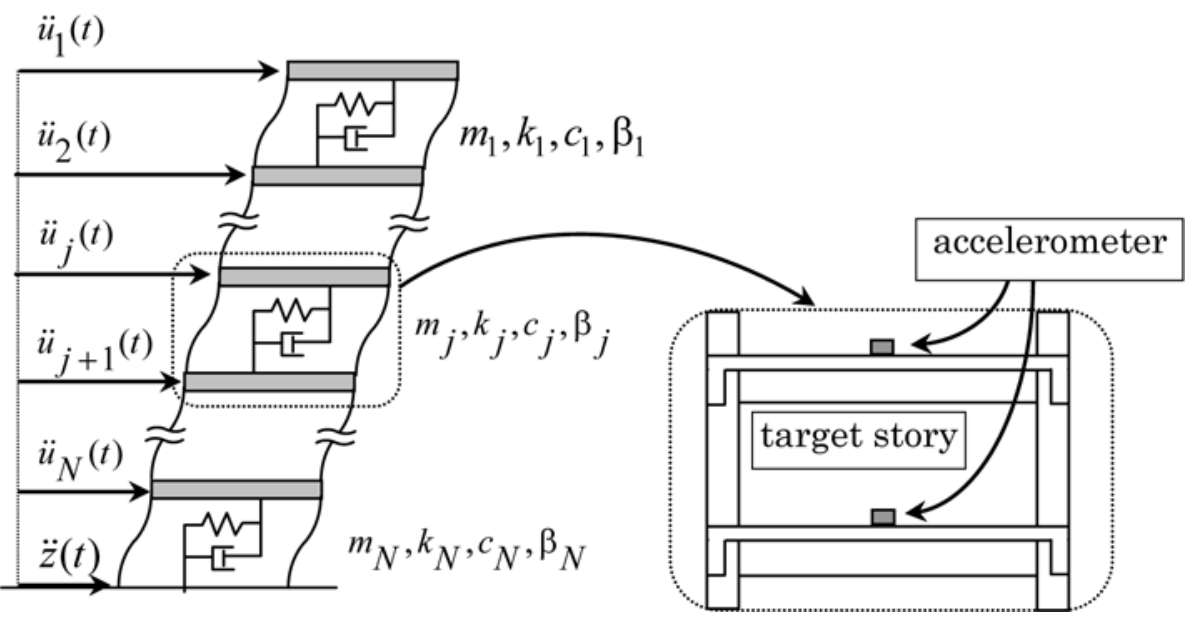

Fig.1 Shear building model and measurement at floors just below and above the target story

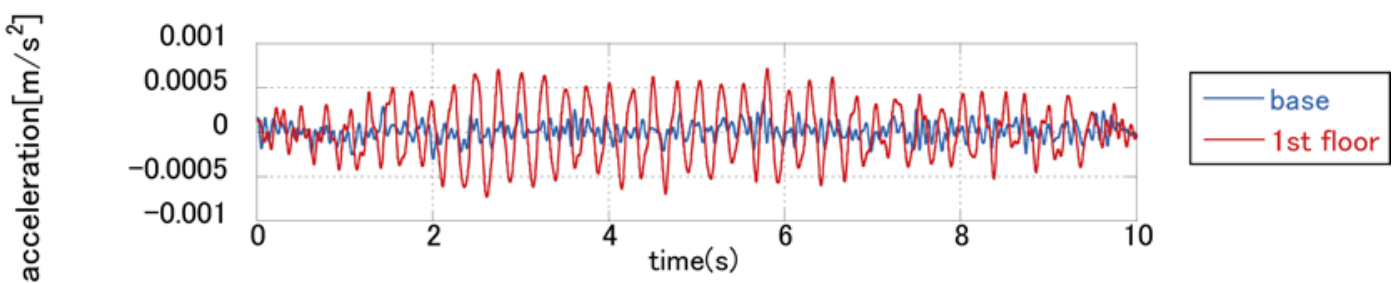

(a) Simulated acceleration without noise

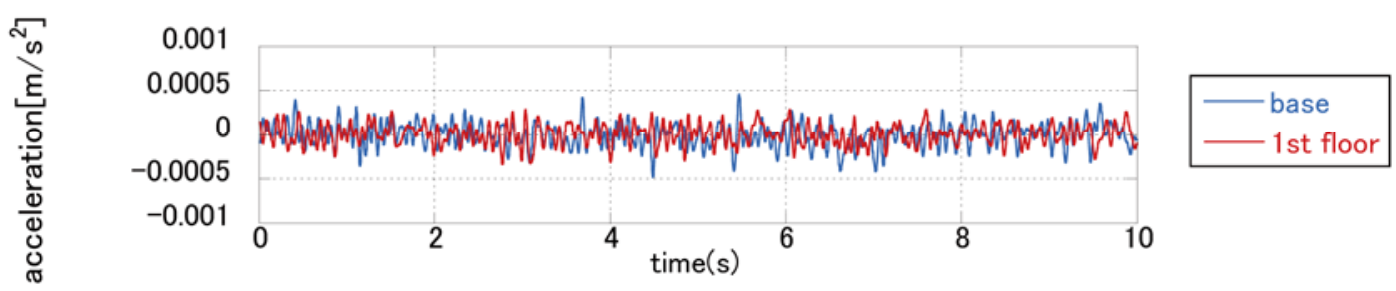

(b) Simulated acceleration of noise

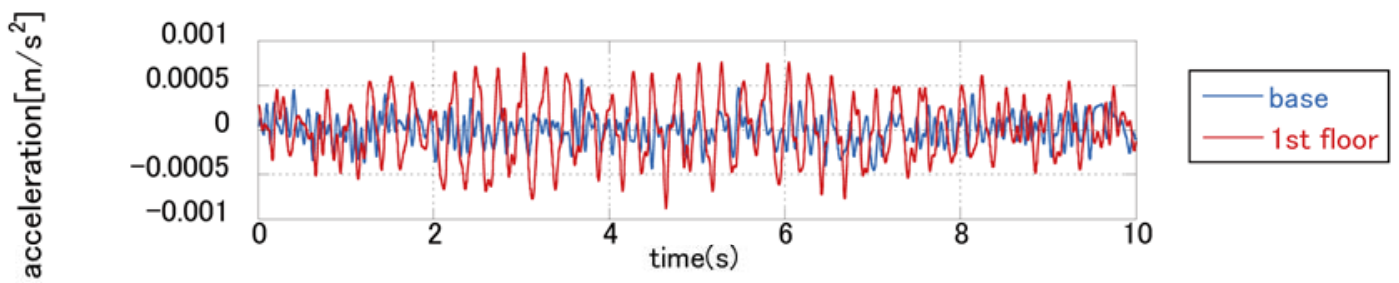

(c) Simulated acceleration including noise

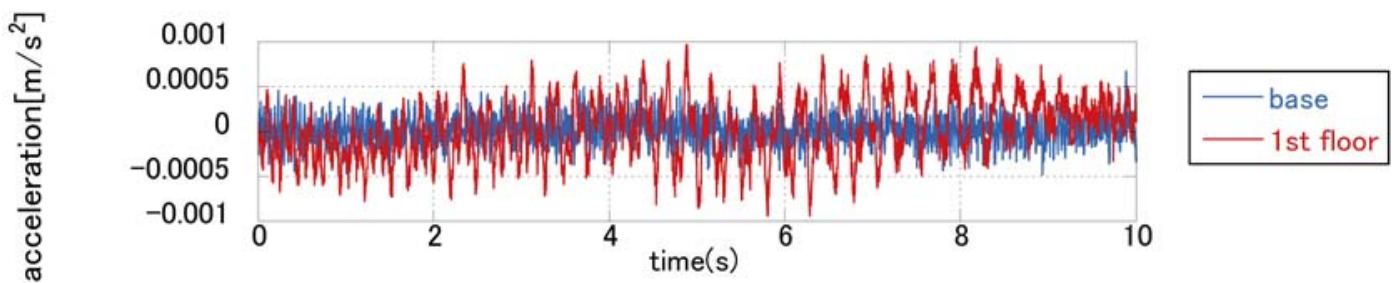

(d) Recorded actual acceleration of microtremor

Fig.2 Simulated and recorded acceleration 


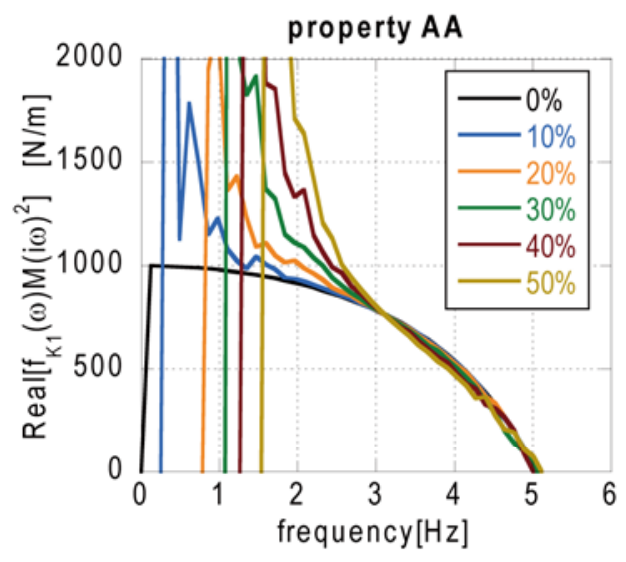

(a) $\operatorname{Re}\left\{f_{K 1}(\omega) M(\mathrm{i} \omega)^{2}\right\}$

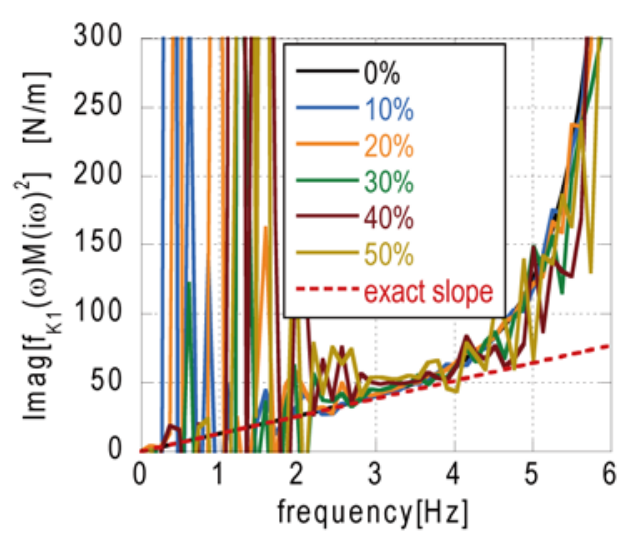

(c) $\operatorname{Im}\left\{f_{K 1}(\omega) M(\mathrm{i} \omega)^{2}\right\}$

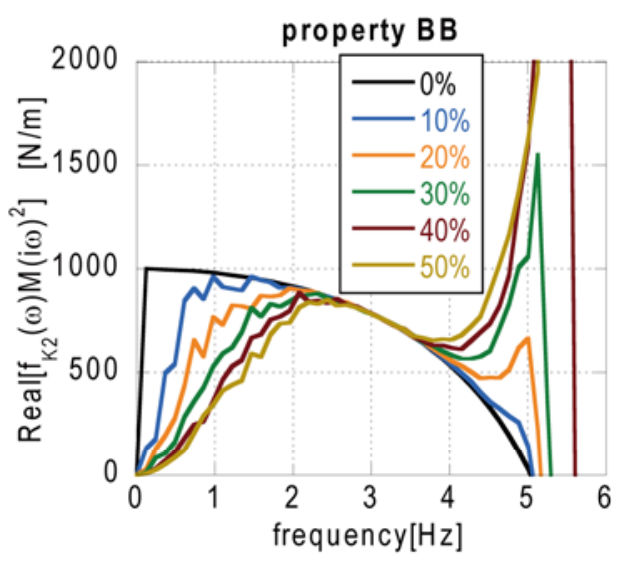

(b) $\operatorname{Re}\left\{f_{K 2}(\omega) M(\mathrm{i} \omega)^{2}\right\}$

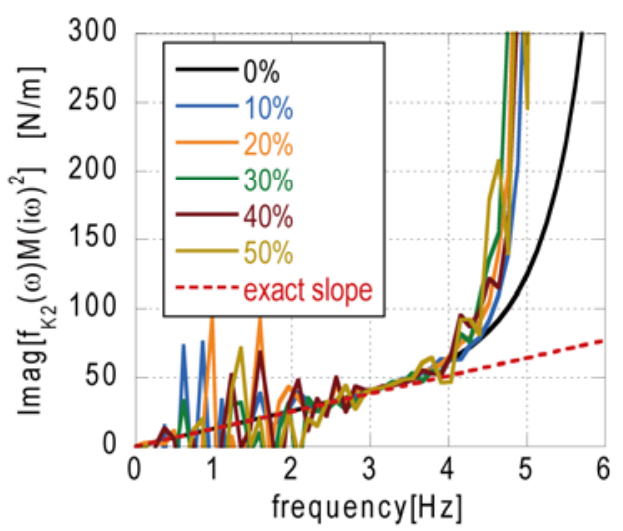

(d) $\operatorname{Im}\left\{f_{K 2}(\omega) M(\mathrm{i} \omega)^{2}\right\}$

Fig.3 Effect of noise level on accuracy of identification

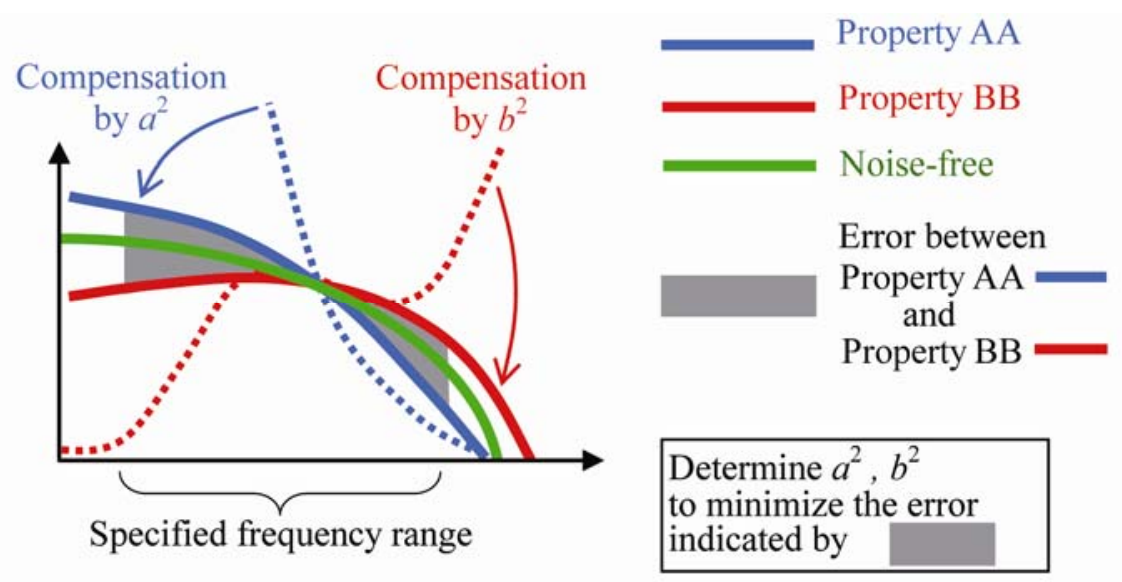

Fig.4 Concept of the proposed noise-bias compensation method 


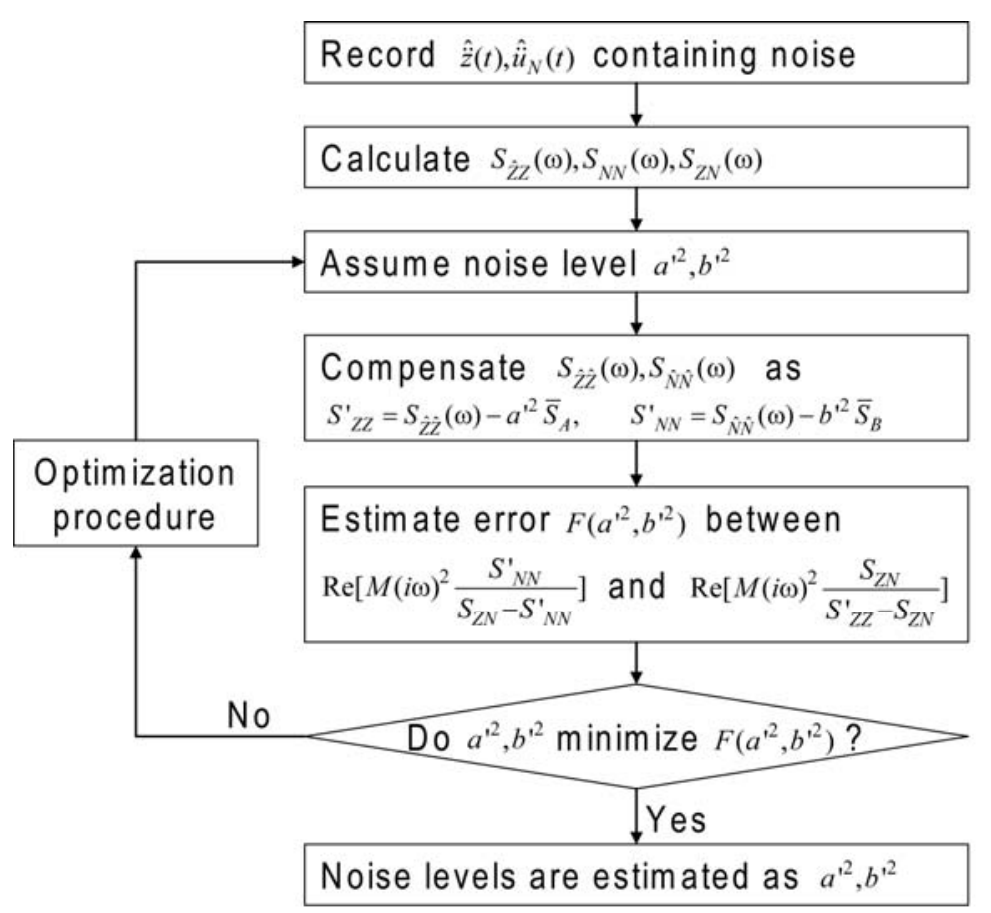

Fig.5 Flow chart of proposed noise-bias compensation method

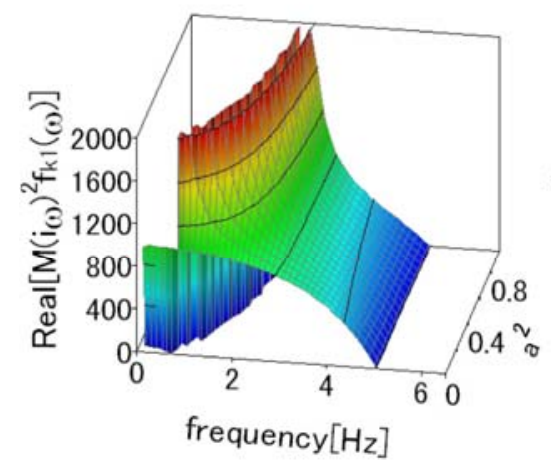

(a) $\operatorname{Re}\left\{\hat{f}_{K 1}(\omega) M(\mathrm{i} \omega)^{2}\right\}$

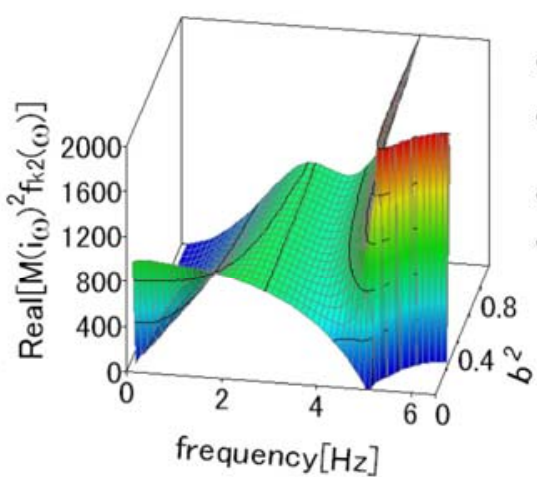

(b) $\operatorname{Re}\left\{\hat{f}_{K 2}(\omega) M(\mathrm{i} \omega)^{2}\right\}$

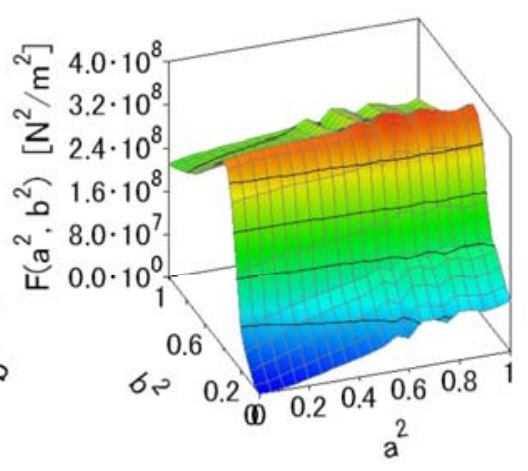

(c) $\hat{F}\left(a^{2}, b^{2}\right)$

Fig.6 Effect of noise level on error function before compensation 


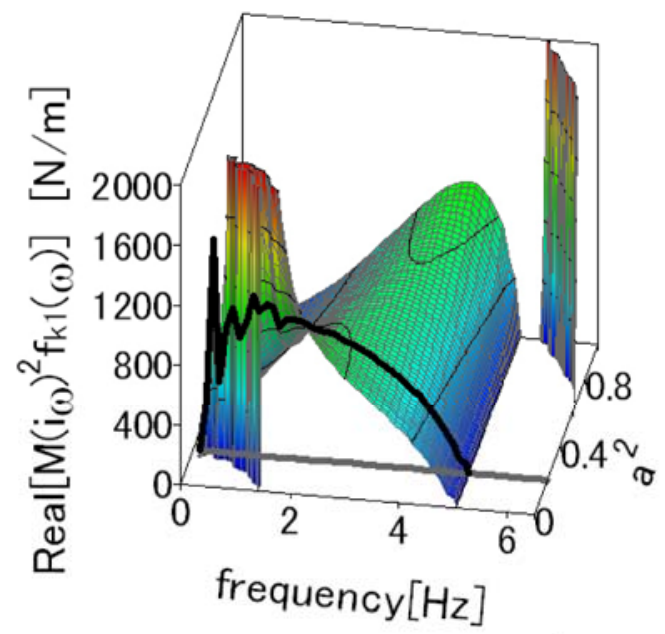

(a) $\operatorname{Re}\left\{f^{\prime}{ }_{K 1}(\omega) M(\mathrm{i} \omega)^{2}\right\}$

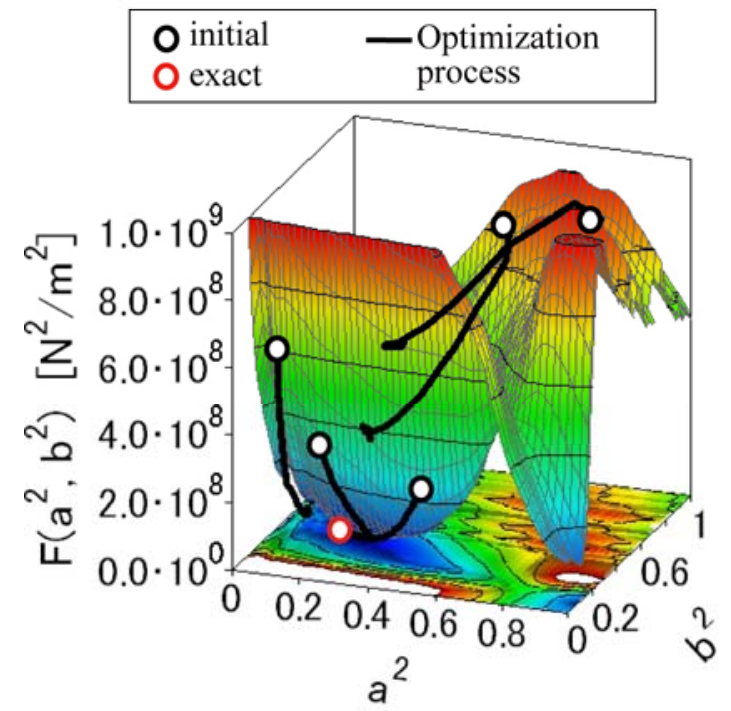

(c) $F\left(a^{\prime 2}, b^{\prime 2}\right)$

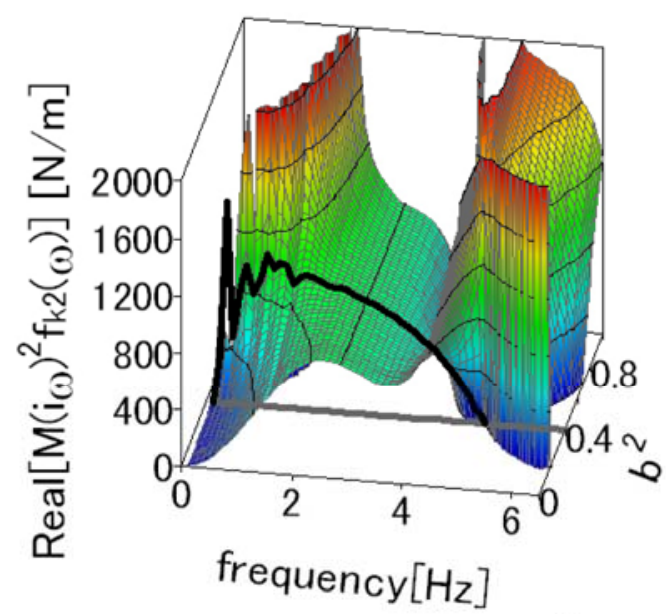

(b) $\operatorname{Re}\left\{f^{\prime}{ }_{K 2}(\omega) M(\mathrm{i} \omega)^{2}\right\}$

Fig.7 Effect of noise level on error function after compensation and optimization process

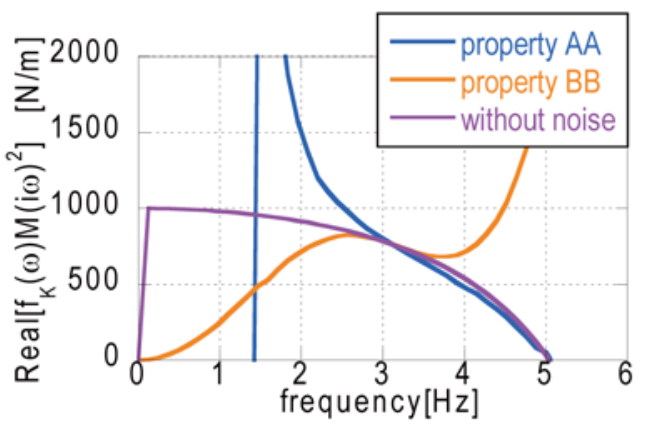

(a) before compensation

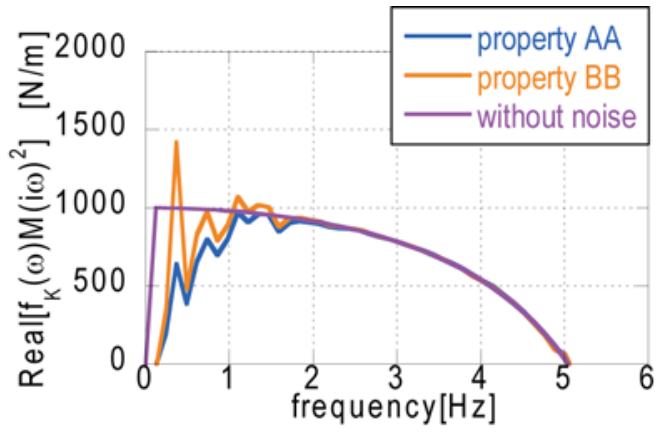

(b) after compensation

Fig.8 Effect of noise-bias compensation on accuracy of identification 


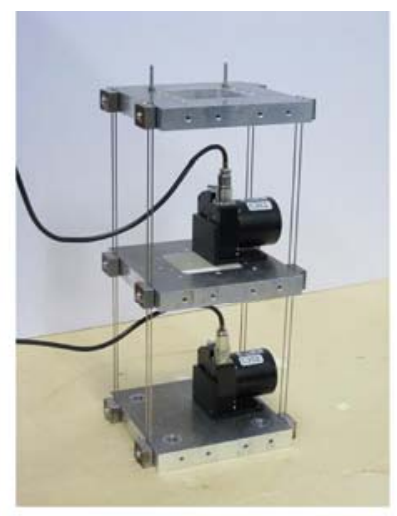

(a) Photo of experimental apparatus

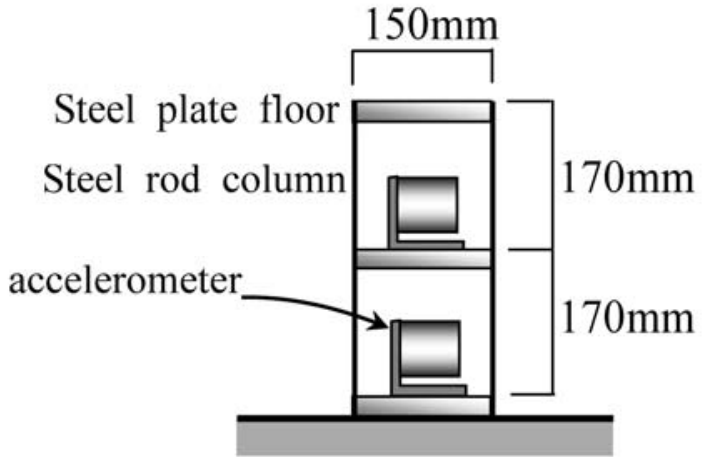

(b) Schematic diagram of experimental set-up

Fig.9 2-story experimental steel frame

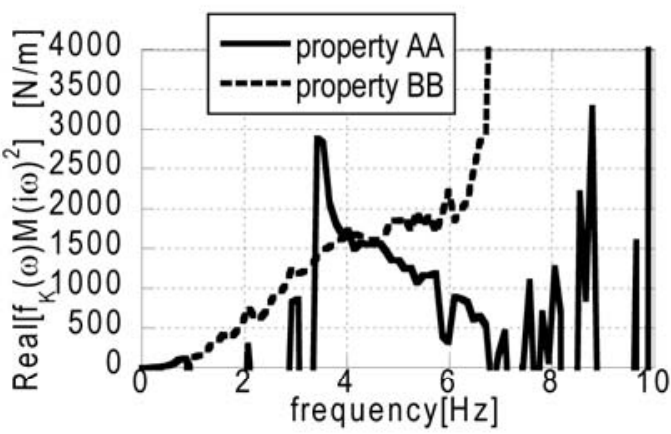

(a) actual data (stiffness)

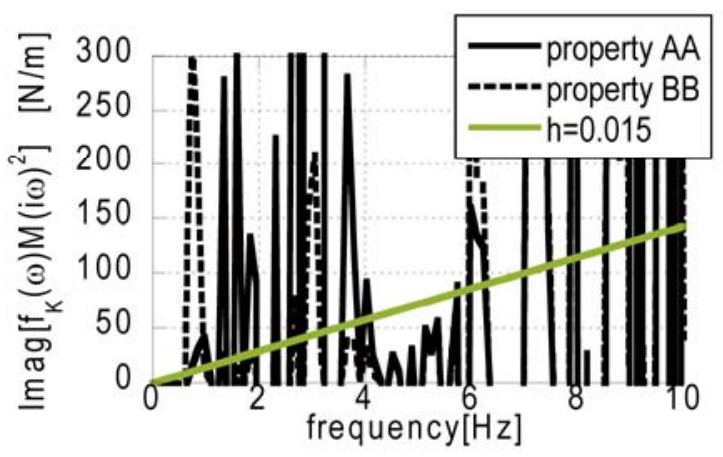

(c) actual data (damping)

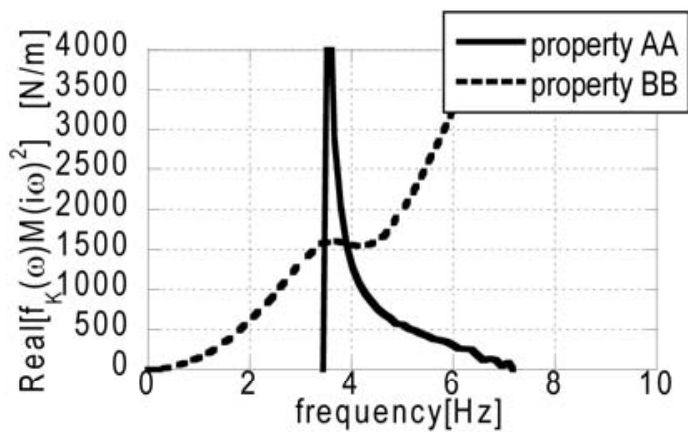

(b) simulation (stiffness)

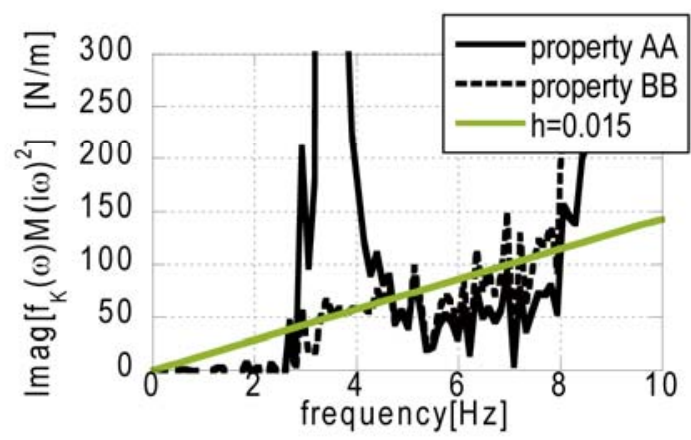

(d) simulation (damping)

Fig.10 Stiffness and damping identification of first story 


\begin{tabular}{|c|c|}
\hline $\begin{array}{l}\text { O initial } \\
\text { O exact }\end{array}$ & -Optimization \\
\hline 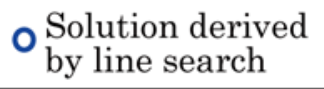 & $\begin{array}{l}\text {.... Direction of } \\
\text { line search }\end{array}$ \\
\hline
\end{tabular}

\begin{tabular}{|c|c|}
\hline $\begin{array}{l}\text { O initial } \\
\text { o exact }\end{array}$ & 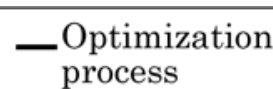 \\
\hline 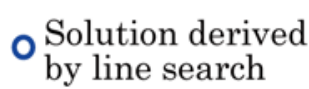 & $\begin{array}{l}\text {.... Direction of } \\
\text { line search }\end{array}$ \\
\hline
\end{tabular}

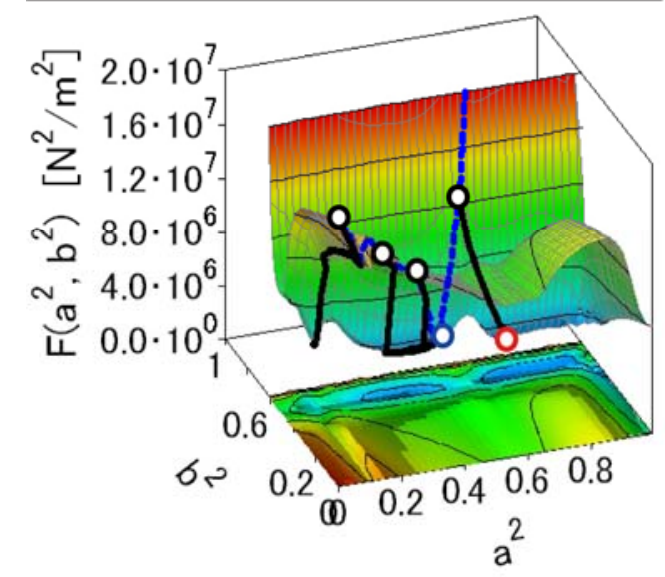

(a) $F\left(a^{\prime 2}, b^{\prime 2}\right)$

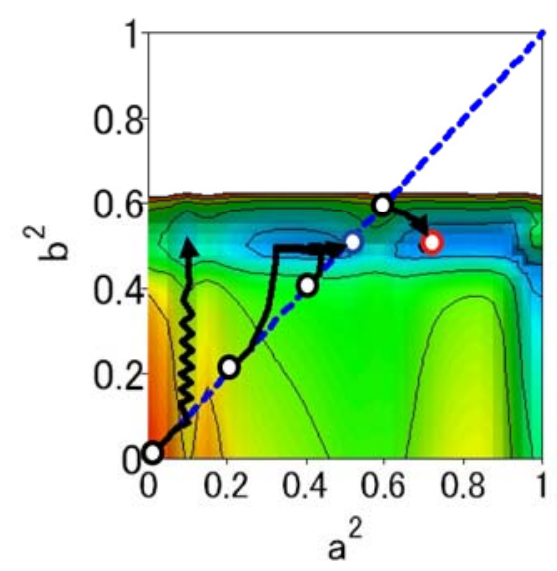

(b) $F\left(a^{\prime 2}, b^{\prime 2}\right)$

Fig.11 Optimization process from various starting points and constrained line $a^{\prime 2}=b^{\prime 2}$ used for line search

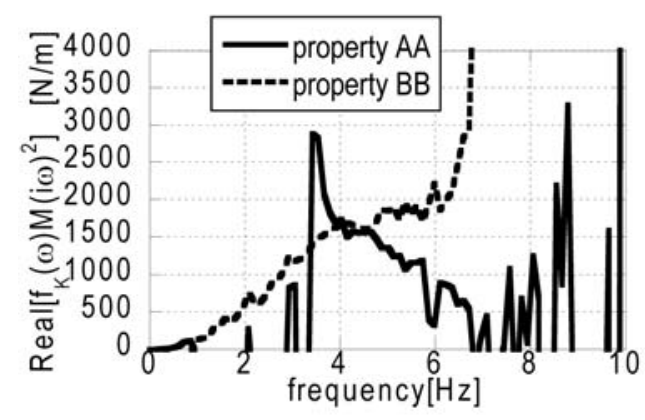

(a) before compensation (stiffness)

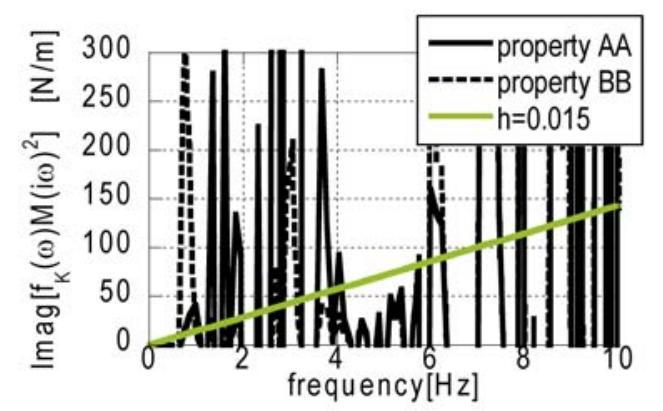

(c) before compensation (damping)

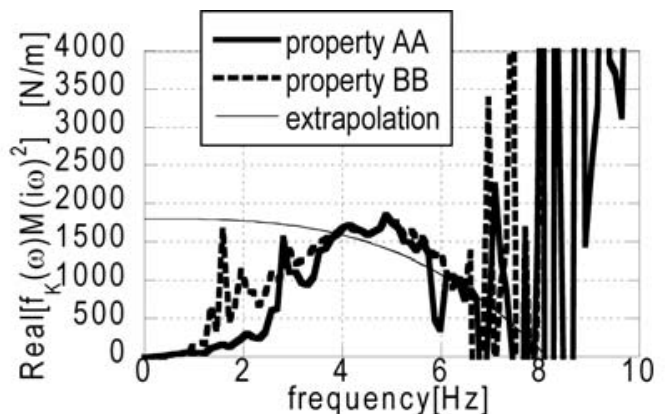

(b) after compensation (stiffness)

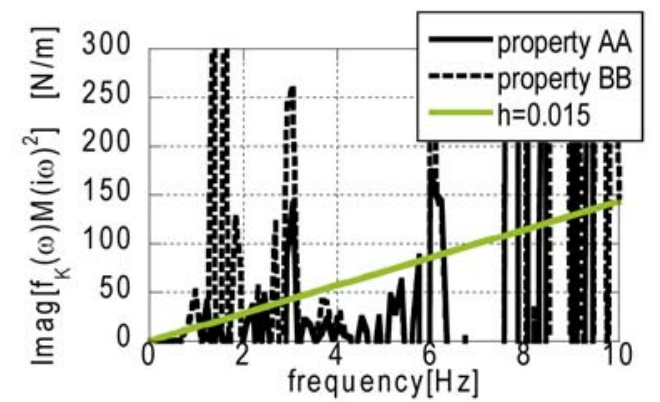

(d) after compensation (damping)

Fig.12 Effect of noise-bias compensation method (first story identification) 